\title{
An Example-based Color Transfer and Style Enhancement Through Locally Neighbor Embedding
}

\author{
Liu Tao, Daiguo Deng, Xuelian Wu and Kun Zeng
}

\begin{abstract}
Image color and tone adjustment are often used for style enhancement in image editing. In this paper we propose a new LLE-based image color transfer and tone enhancement method. Our target is to find the implicit color mapping rule between example image-pairs, then apply it to new image. Given a pair of images with the same size and theme but different color styles, we first divide them into lots of small patches and learn the implicit relationships among those patches using locally linear embedding(LLE). Then we impose the learned transfer pattern on input image, and take some extra processing techniques to make output image more natural. We apply our method for image color transfer andcolor images enhancement in these experiments, and the results show that our approach is effective.
\end{abstract}

KeywordsColor transfer • Tone enhancement • Locally linear embedding

\section{Introduction}

Image styles are usually represented by image colors and tones. In the field of photography, a lot of works use large number of colors to generate special image styles and visual effects. in the field of art and design, people commonly do color editing and rendering to enhance the image display effects. But for those image editing workers, it is a tedious and boring task to match such large number of colors for images. If we are able to directly take good effect images as examples and learn colors and tones from them, it would be a meaningful work. Meanwhile, traditional algorithms do not perform very well in accurate local color transfer and little work is from a learning perspective.

Liu Tao, Daiguo Deng, Xuelian Wu

National Engineering Research Center of Digital Life,

State-Province Joint Laboratory of Digital Home Interactive Applications,

School of Information Science \& Technology, Sun Yat-sen University, Guangzhou 510006, China

Kun Zeng $(\bowtie)$

School of Software, Sun Yat-sen University, Guangzhou 510006, China

email: zengkun@gmail.com 
This paper proposes an example based learning algorithm which has a good effect on both image tone enhancement and accurate local image color transfer. We first divide example images into lots of patches and learn the implicit relationships between them with locally linear embedding(LLE); then apply the learned pattern to new images and finally take a gradient-preserving work to get our output image. Pipeline in details could be referred in Figure 1.

Main contribution of our work is that we build an image color transfer and enhancement model based on learning algorithms. Support vector machine (SVM), LLE or other learning algorithms are supported under this model. Specifically with our learning method, we have a very good performance onboth image tone enhancement and local image color transfer.

Other parts of the paper are as following. Chapter 2 summarizes existing manifold learning and colortransfer methods. Chapter 3 introduces LLE learning model and procedures.Chapter 4 presents experiments results and makes comparisons with existing methods. Chapter 5 makes a conclusion and discusses about future work.

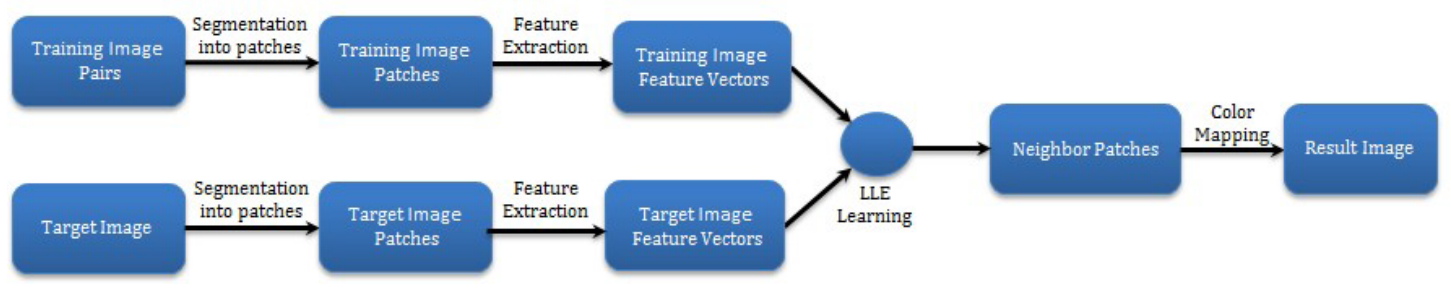

Fig. 1The pipeline of our LLE transfer approach.

\section{Related Work}

\subsection{Color Transfer}

Reinhard et al.[1] first propose a global image color transfer method. They usel $\propto \beta$ color space which has little correlation between its three channels. And they consider all pixels present a Gaussian distribution in each channel. The mean value of each channel in target image substitutes the corresponding one in source image. All pixels are modified according to the mean and variance. They do a good job with this approach that result images look rather smooth and natural under condition ofno post-processing after transferring, and it runs at high efficiency. The weakness of this approach is that natural image pixels present not just a simple Gaussian distribution, andthe result image wouldscatter local concentrating colors to global instead of corresponding relative local areas which means that it has a poor performance in local image color transfer.

On this basis, Tai et al.[2] propose an algorithm that uses EM(Expectation Maximization) to segment images under consideration of probabilities. They 
assemble source and reference image with K-means to build a Gaussian Mixture Model(GMM) respectively. Then match similar Gaussian distribution pairs in the source and reference image to transfer colors. This approach produces appropriate number of Gaussian distribution model and fit the image color distribution comparatively well. But for the algorithm of improved EM-based probability segmentation is complex and GMM-building is time-consuming, it's not good for big data process.

Pitiéet al.[3] achieve global image color transfer with $\mathrm{N}$-dimensional histogram matching instead of considering image color as a Gaussian distribution or GMM. They project color distributions to different axes, then map color histogram between those two images. Repeat this step to result to a convergence at last. Their approach transfers most of color information in target image to source image rather well, but lead tograin artifacts at the same time. Forpart of local information is losing in the procedure, some post processing steps are necessary. As last, computing complexity adds up.

Huang et al.[4] 2009 propose a novel image color transfer algorithm to make use of a grayscale picture and a little amount of colorful feature points called landmark points. They achieveto recovera color image with the grayscale picture and landmark pixels. Thus, color transfer could be considered a procedure ofexchanging source image's landmark pixels with reference image's. This method is highly effective, but has a weakness of color rendering confusion.

$\mathrm{Li}$ et al.[5] employ neighbor embedding to colorize grayscale images. They first segment images into patches and present them with feature vectors. Then get reconstruction coefficient array through computing source image patch's linear express by neighbor fields. Finally construct the output image with the coefficient array and target image. This method is effective but limited to grayscale image coloring.

Wang et al.[6] propose an example-based image color and tone style enhancement method, which has a widely application. They take a pair of images of same theme and size, then extract features of pixels at same position and find implicit mapping relationships between them, finally impose the relationship on an input image. Much extra efforts are needed such as luminance gradient preserving and tone optimization. The approach is effective, but complex and time-consuming.

\subsection{Manifold Learning}

Suppose that data is uniformly sampled in low-dimensional manifold from a high-dimensional Euclidean space, manifold learning is the low-dimensional manifold structure recovery from high-dimensional sampling data, namely, to find a low-dimensional manifold in high-dimensional space, and seekingthe corresponding embedding mapping to achieve dimensionality reduction or data visual- ization. It is 
about finding the essence of things from the observed phenomenon, and mining data inherent laws.

Manifold learningis divided into linear manifold learning and nonlinear manifold learning. Nonlinear manifold learning includesisometric feature mapping (Isomap)[7], Laplacian Embedding(LE)[8], Locally Linear Embedding (LLE)[9]; Linear methods is linear expansion of the non-linear methods, such as local preserving projection(LPP)[10] and neighbor preserving embedding (NPE)[11] and so on.

\section{LLE Transfer}

\subsection{Locally Linear Embedding}

Locally linear embedding(LLE) employs local linearization method, mapping the high-dimensional, non-linear data to a global low-dimensional space by building a local linear hyper-plane to maintain the translation and rotation invariance of neighbor points set's structure. LLE is used for nonlinear dimensionality reduction, which extracts the essential characteristic of high-dimensional data and keep the global geometric properties. Besides, lower computing complexity makes it applicable to many dimensionality reduction areas.

LLE divides the whole data space into $n$ local areas. Data in each area can be linearly expressed with its neighbors. For only neighbors participating in data reconstruction, low-dimensional manifold can be retrieved from their geometric information. The three key points of LLE are as follows:

1. For each point in high dimension space, compute its $\mathrm{k}$ nearest neighbor points according to Euclidean distance.

2. Calculate the reconstruction weights for $\mathrm{k}$ nearest neighbor points in accordance with the principles of the Least Squares.

3. Construct the low-dimensional data space with the above weights and maintain the local linear structure as in high-dimension space as much as possible.

\subsection{Our LLE Transfer}

In our LLE transfer method, training images and input image will be first divided into same-sized and overlapped patches. Local geometric features are kept through reconstruction of $k$ nearest image patches' feature vectors.

As in Figure 1, for an image patch $\boldsymbol{x}_{t}$ in training target image, first we find the $k$ nearest image patches $\left(\boldsymbol{x}_{s}^{i}, i=1 \ldots k\right.$. $)$ in source image by feature vector'Euclidean distance and compute reconstruction weight $\boldsymbol{w}_{i}$ with the Least Squares method. 
Second, we compute image patch $\boldsymbol{Y}_{t}$ in output image with $\boldsymbol{x}_{s}^{j}(j=$ $1 \ldots k)$ corresponding patches $\boldsymbol{y}_{s}^{j}$ in training images and the reconstruction weight $\boldsymbol{w}_{i}$. The pseudo code of the algorithm is as follows:

1.Initialization of data sets: training images $\boldsymbol{X}_{s}$ and $\boldsymbol{X}_{t}$.

2.Repeat for each patch $\boldsymbol{x}_{t}^{q}$ in training target image $\boldsymbol{X}_{t}$ :

(a) Find set $N_{q}$ : the $k$ nearest neighbors of $\boldsymbol{x}_{t}^{q}$ in $\boldsymbol{X}_{s}$.

(b) Compute reconstruction weights of neighbors that minimize the Error forreconstructing $\boldsymbol{x}_{t}^{q}$.

(c) Computetarget result embedding $\boldsymbol{y}_{t}^{q}$ using appropriateresult features of the knearestneigh-

borsin image $\boldsymbol{Y}_{s}$ and the reconstruction weights.

3.Construct the target result image $\boldsymbol{Y}_{t}$ by enforcing local compatibility and smoothness constrain between adjacent patches obtained in step 2(c).

In Step2(a), we find the $k$ nearest neighbors by compute feature vector's Euclidean distance. Then calculate the minimum reconstructing error weight with the Least Squares method:

$$
\boldsymbol{\varepsilon}^{q}=\min \sum_{t}\left\|\boldsymbol{x}_{t}^{q}-\sum_{x_{s}^{p} \in N_{q}} w_{q p} \boldsymbol{x}_{s}^{p}\right\|^{2}
$$

Subject to

$$
\begin{aligned}
& \sum_{x_{s}^{p} \in N_{q}} w_{q p}=1 \\
& w_{q p}=0, \text { for } \forall x_{s}^{p} \notin N_{q}
\end{aligned}
$$

Optimal solution of Eq(1) is also easily found in Reference [9]. In Step 2(c), $\boldsymbol{y}_{t}^{q}$ is counted by the reconstructing coefficient $\boldsymbol{w}_{q}$ and $\boldsymbol{y}_{s}^{q}$, the feature vector of target source image's patch that $\boldsymbol{x}_{s}^{q}$ corresponds to:

$$
\boldsymbol{y}_{t}^{q}=\sum_{x_{s}^{p} \in N_{q}} w_{q p} \boldsymbol{y}_{s}^{p}
$$

To achieve a visual satisfied result, we propose a guided smoothing model based on the guided filter to suppress artifacts[12]. 


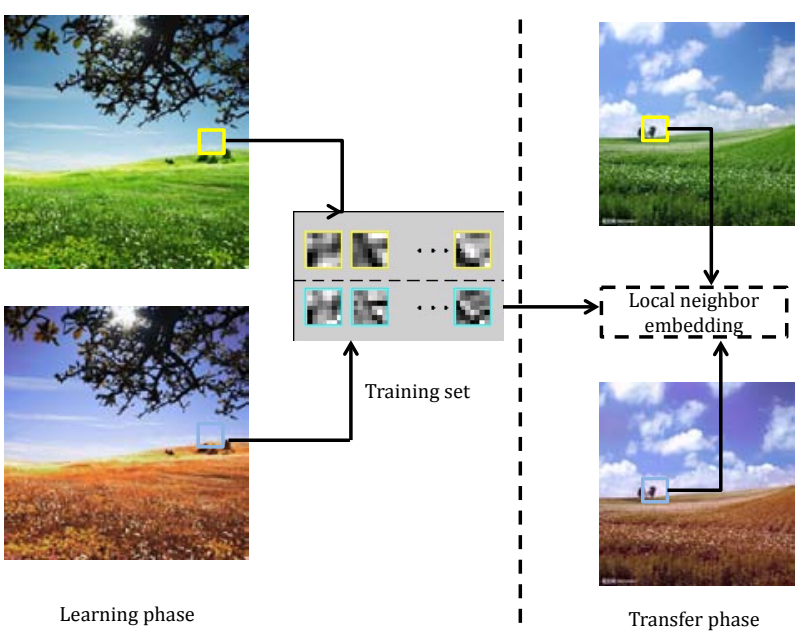

Fig. 2Localneighbor embedding and learning process.Left two are example images, we divide them into patches and learn the implicit relationships. In transfer phase, we utilize this relationship and an input image to get our result image.

\subsection{Feature representation}

Feature extraction is a very important question which directly affects experiments results. Our feature representation treats $3 \times 3$ pixel patch as a unit and operates in lab color space instead of RGB because of the least correlation between different channels. Thus, when mapping channel values into feature vector, different dimensions correlates least too. First we take pixels' three channel value to compose a 27 dimensional vector. Then, for $\mathrm{L}$ channel we count its $\mathrm{x}$ axis and $\mathrm{y}$ axis's gradient value respectively so that pixel patch matches more accurate under the consideration of gradient information. For channel a and b, it's not required to take their gradient values based on such a vision fact: human eyes are more sensitive to luminance changes than color differences[13]. So our final feature vector is represented by a 45 dimensional vector.

\section{Experiments and Results}

All the algorithm are coded in Matlab and are run onPC with Dual-Core 2.2GHz Intel Pentium 4 processor CPU. In Fig.3, the first row shows we learn the transfer pattern of more brilliant and yellow tone from the training images, as a result, leaves in the fourth column is obvious more lightful and vivid than the third; the second row 
shows we learn the red tone style transferfrom the training images, finally, our output image appears a more attractive red tone style and a warning color atmosphere.

In Fig.4, the color of sky and grass transfers well in our learning algorithm. In first row, gray sky and yellow grassland in reference image and output imageare almost identical. While with Reinhard's method, grass' color remains green which seems it didn't transfer from reference image at all, and sky's color is almost like the average color of source image and reference image, instead of reference image's color. In Pitié's approach,the sky's color distorts, and the grass's color is over matching to take on a strong yellow color which is different with the reference's too. In second row, Reinhard's approach performs better than in first row, but local color transfer is not so accurate, the yellow color in reference's grassland scatters to sky already, and Pitié'sway to transfer the local color is also not as well as ours and color in sky occur huge grain artifacts.
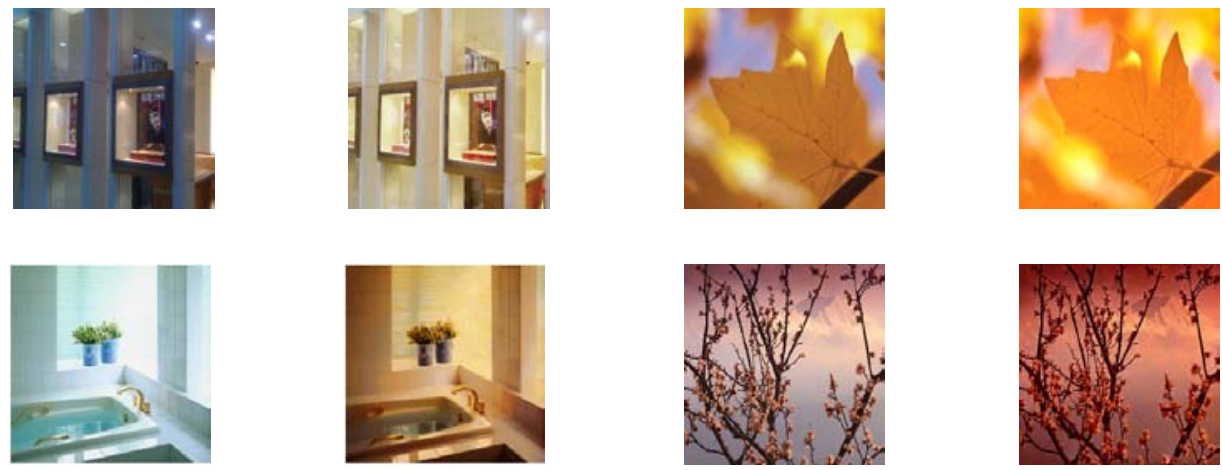

Fig.3Image color enhancement result. Left top is a training image pair. Right top is a target image and output image that results from our LLE learning algorithm. Pictures in second line arrange the same as in first line.

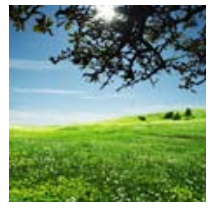

(a) source

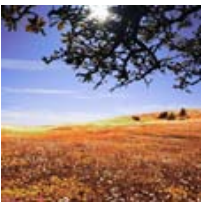

(b) reference

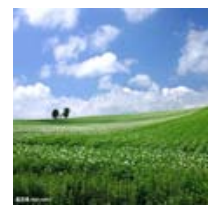

(c) target

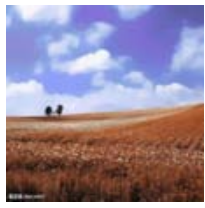

(d) our result

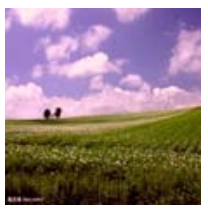

(e) Reinhard

(f) Pitié 
Fig. 4Image color transfer. The first and second column is source image and reference image respectively. The fourth column is our result image. The last two columns correspond to result images of Reinhard and Pitié's respectively.

In Fig. 5, we draw color distributions in 1D and 2D spaces to intuitivelycompare between different methods. Pitié'smatches the reference image's color distribution best. Our method's resultis similar to reference image's, while there is a big gap between Reinhard's result image and reference image. But overall our result image is most close to the reference image.

As for the computing complexity of our algorithm, Eq(1) is finally transformed to solve a least-squares problem. Optimal solution is to compute a linear system of equations which could be referred in Reference [9] for details. Typically, for $512 \times 512$ pixels image and $k=12$, our method takes about 413 seconds.

Parameters in our approach mainly compose of feature vectors, overlapping factor and nearest neighbor count $k$. Here in our experiments, we take a 3x3 pixel patch. If a patch is too large, high dimensional vector will cause more complex matrix computing, on the other hands, if too small patch, it would lead to low-quality patch matching. Overlapping factor is 2, and we take average value of adjacent patches on overlapping areas to make our result picture smoother. Nearest neighbor number is set to 12 , which is the best during our seriesexperiments test.
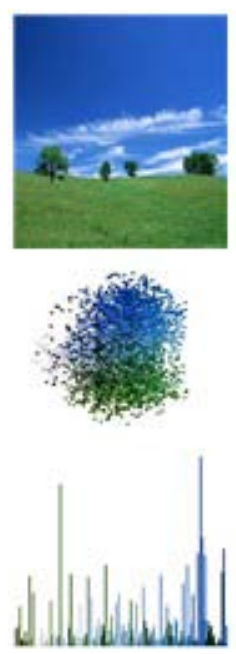

(a) source
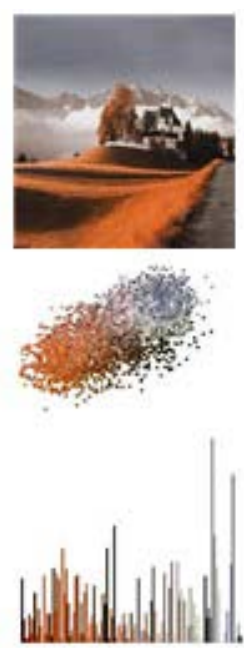

(b) reference
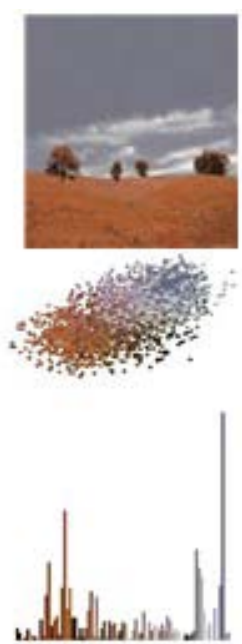

(c) our result
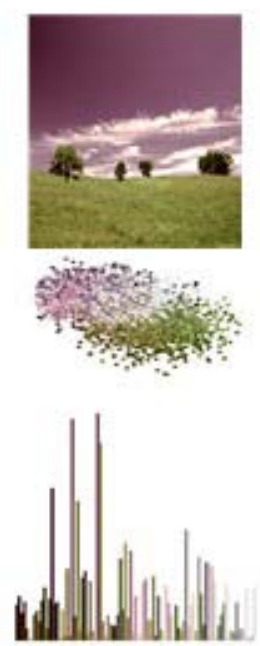

(d) Reinhard
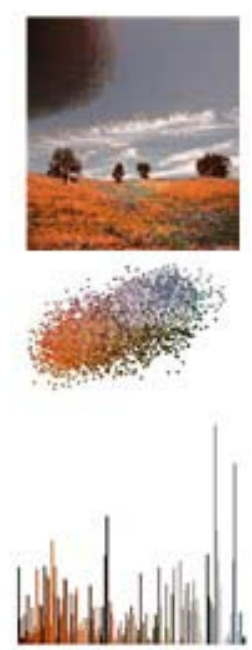

(e) Pitié

Fig. 5Image color distribution comparisons. Pitié'sapproach has the same color distribution with reference image's. Our method's distribution is similar to reference image's. Reinhard'sresult is the worst. But overallour method performs best without grain artifacts comparing to Pitié and Reinhard's. 


\section{Conclusions}

This paper proposes a new learning-based image color transfer and tone enhancement method. The approach finds the implicit transfer pattern between the training image pair, and applies it for a new input image to present our desired result. It is effective and the process is simpler than the svm-based methods in Reference[6]. The weakness of this approach is that the two pictures of different scenarios should have some kind of similarity so that patches in target image could always find appropriate linear expression with patches in source image. Our method doesn't work well if the two pictures are totally different in details. The problem is under consideration in our future work, and we will try other learning methods and do more jobs on feature presentation.

AcknowledgementsThis research is supported by NSFC-Guangdong Joint Fund (U1135003, U0935004), the National Key Technology R\&D Program (2011BAH27B01, 2011BHA16B08). Thanks to Guangyuan Huang for the kind suggestions on paper writing.

\section{References}

1. Erik Reinhard, Michael Ashikhmin, Bruce Gooch, andPeter Shirley.Color transfer between images. IEEEComputer Graphics and Applications, vol. 21, no. 5, pp.34-41, 2001.

2. Yu-Wing Tai, JiayaJia, and Chi-Keung Tang.Localcolor transfer via probabilistic segmentation byexpectation-maximization. CVPR, 2005, pp. 747-754.

3. François Pitié, Anil C. Kokaram, and RozennDahyot.N-dimensional probablility density function transferand its application to colour transfer.ICCV, 2005,pp. 1434-1439.

4. T.W. Huang and H.T. Chen.Landmark-based sparsecolor representations for color transfer.ICCV, 2009,pp. 199-204.

5. J. Li, P. Hao, and C. Zhang.Transferring colors tograyscale images by locally linear embedding.Proc.British Machine Vision Conf.(BMVC), Leeds, UK, 2008,pp. 835-844.

6. B. Wang, Y. Yu, and Y.Q. Xu.Example-based imagecolor and tone style enhancement.ACM TOG, vol. 30,pp. 4, 2011.

7. J.B. Tenenbaum, V. De Silva, and J.C. Langford.Aglobal geometric framework for nonlinear dimensionalityreduction. Science, vol. 290, no. 5500, pp. 2319-2323, 2000.

8. M. Belkin and P. Niyogi.Laplacianeigenmaps for dimensionalityreduction and data representtation.Neuralcomputation, vol. 15, no. 6, pp. 1373-1396, 2003.

9. Sam T. Roweis and Lawrence K. Saul.Nonlinear dimensionalityreduction by locally linear embedding.Science, vol. 290, no. 5500, pp. 2323-2326, December2000.

10. P. Niyogi.Locality preserving projections.Advancesin neural information processing systems, vol. 16, pp.153-160, 2004. 
11. X. He, D. Cai, S. Yan, and H.J. Zhang.Neighborhoodpreserving embedding.Computer Vision, 2005.ICCV 2005. Tenth IEEE International Conference onIEEE, 2005, vol. 2, pp. 1208-1213.

12. K. He, J. Sun, and X. Tang.Guided image filtering.Computer Vision-ECCV 2010, pp. 1-14, 2010.

13. B.A. Wandell.Book rvw: Foundations of vision. Bybrian a. wandell.Journal ofElectronic Imaging, vol. 5, no. 1, pp. 107-107, 1996. 